H. ITO

KODAI MATH. J.

3 (1980), 442-456

\title{
LONG PERIODIC SOLUTIONS NEAR AN EQUILIBRIUM IN GENERAL RESONANCE CASES
}

\author{
By HidekAZU ITO
}

\section{Introduction}

In this paper, we study the existence of periodic solutions of a system of ordinary differential equations

$$
x=f(x)
$$

near an equilibrium solution. Here $x$ and $f(x)$ are real $m$-dimensional column vectors with entries $x_{k}$ and $f_{k}(x)(k=1, \cdots, m)$ respectively, and the dot indicates differentiation with respect to the real independent variable $t$. We may take the equilibrium solution to be the origin $x=0$, and then we assume that the $f_{k}(x)$ $(k=1, \cdots, m)$ are $C^{1}$ functions of $x_{1}, \cdots, x_{m}$ in a neighborhood of the origin.

For the study of periodic solutions of (1.1) near the origin, the eigenvalues of $C=f_{x}(0)$ are crucial. Here $f_{x}(0)$ denotes the Jacobian matrix of $f(x)$ at $x=0$, and throughout this paper we assume that $C$ is non-singular. As is well known, the presence of purely imaginary eigenvalues of $C$ is necessary for (1.1) to have periodic solutions near the origin, but not sufficient. Let $C$ have eigenvalues $\lambda_{1}, \cdots, \lambda_{m}$ such that $\lambda_{1}$ is purely imaginary and $\lambda_{2}=\bar{\lambda}_{1}$. Then the well known Liapunov's Theorem guarantees the existence of a one-parameter family of periodic solutions with primitive periods near $2 \pi /\left|\lambda_{1}\right|$ under two assumptions. One of them is that none of the $m-2$ quotients $\lambda_{k} / \lambda_{1}(k=3, \cdots, m)$ is an integer (nonresonance condition), and the other is that (1.1) possesses an integral whose Hessian at $x=0$ restricted to $E_{1}$ is nondegenerate, where $E_{1}$ is the real two-dimensional (generalized) eigenspace corresponding to $\lambda_{1}$ and $\lambda_{2}$ (see [4]).

In this paper, we consider the cases when the above first assumption is violated, that is resonance cases. Then, we can assume that $C$ has eigenvalues $\lambda_{1}, \cdots, \lambda_{m}$ satisfying the following conditions for an integer $r(\geqq 2)$ :

$$
[\mathrm{A} .1]\left\{\begin{array}{c}
(\mathrm{i}) \quad \begin{array}{l}
\lambda_{k}=\imath \sigma_{k} \\
\text { imaginary eigenvalues such that }
\end{array} \\
\sigma_{k}=n_{k} \sigma_{1}\left(\sigma_{1}>0\right), \quad 1=n_{1} \leqq n_{2} \leqq \cdots \leqq n_{r},
\end{array}\right.
$$

where $n_{k}(k=1, \cdots, r)$ are positive integers.

(ii) no $\lambda_{k+2 r}(k=1, \cdots, s)$ is an integer multiple of $\lambda_{1}$, where $s=m-2 r$.

Received November 6, 1979 
Here, of course, the condition (ii) is eliminated if $2 r=m$.

We consider the resonance cases under this assumption [A.1], and the aim of this paper is to establish the existence of periodic solutions of (1.1) near $x=0$ whose primitive periods are close to $2 \pi / \sigma_{1}$. To this end, it is a fundamental assumption that (1.1) possesses an integral. But simple examples show (see [2], [3], [6]), such periodic solutions do not necessarily exist even if (1.1) possesses an integral with nondegenerate Hessian at $x=0$. We wish to accomplish our purpose by imposing the suitable conditions on nonlinear terms of $f(x)$. In this direction, several authors $([1],[4],[5])$ have dealt with resonance cases when $r=2$ in [A.1] mainly for Hamiltonian system

$$
\dot{x}_{k}=\frac{\partial H}{\partial x_{k+m}}, \quad \dot{x}_{k+m}=-\frac{\partial H}{\partial x_{k}} \quad(k=1, \cdots, m) .
$$

Here it is noted that Hamiltonian function $H(x)$ is an integral for (1.2). The results obtained there can be stated in terms of the coefficients in normalized Hamiltonian function. Among their results, it is remarkable that if $n_{2} \geqq 4$, the existence of such periodic solutions is guaranteed by adding a certain condition on the coefficients of fourth order terms of Hamiltonian function in Birkhoff normal form.

In the present paper, we generalize this result for the system (1.1) with an integral in resonance cases for arbitrary $r$. To this end, we must bring the system (1.1) into a certain normal form by means of a suitable change of variables. In the next section, we will show that this is possible under the following assumption on $r-1$ integers $n_{k}(k=2, \cdots, r)$ :

$$
\sum_{k=1}^{r} \jmath_{k} n_{k} \neq 0 \quad \text { for all integer valued vectors }\left(\jmath_{1}, \cdots, \jmath_{r}\right)
$$

$$
\text { with } 1 \leqq \sum_{k=1}^{r}\left|j_{k}\right| \leqq 4 \text {. }
$$

We note here that this assumption means $n_{2} \geqq 4$ when $r=2$. This normal form will be defined there without assuming that (1.1) possesses an integral, and then we shall study this normal form under the existence of an integral. Through the normal form, our result will be stated in section 3 .

It is to be noted that, by the assumption [A.2], $\lambda_{1}, \cdots, \lambda_{2 r}$ are all distinct from one another. Then, since non-resonance condition of Liapunov's Theorem is fulfilled for $\lambda_{r}=\bar{\lambda}_{2 r}$, there exist periodic solutions with primitive periods near $2 \pi / \sigma_{r}$ provided that (1.1) possesses an available integral. In this sense, what is discussed here is the existence of "long" periodic solutions near $x=0$.

The author would like to express his sincere gratitude to Professor Toshihiko Nishimoto and Professor Yoshikazu Hirasawa for their constant encouragements and valuable advices, and to Dr. Haruki Yamada for his helpful comments and suggestions. 


\section{Normal Form}

This section is devoted to the study of a normal form for (1.1). The main purpose is to show that the system (1.1) is taken into the normal form under the assumptions [A.1] and [A.2]. This will be accomplished by proving the following lemma, in which the normal form is defined by the system (2.2) with (2.3) and (2.4).

LEMMA 1. Consider the system (1.1) near the equilibrium $x=0$. Let $f(x)$ be of class $C^{4}$ in a neighborhood of $x=0$. Assume that the eigenvalues of $C=f_{x}(0)$ satisfy the assumptions [A.1] and [A.2]. Then there exists a real analytic substitution

$$
x=\phi(u, v), \quad(u, v)=\left(u_{1}, \cdots, u_{2 r}, v_{1}, \cdots, v_{s}\right)
$$

which takes the system (1.1) into the following normal form:

$$
\begin{gathered}
\dot{u}_{k}=\sigma_{k} u_{k+r}+P_{k}(u) u_{k}+Q_{k}(u) u_{k+r}+U_{k}(u, v), \\
\dot{u}_{k+r}=-\sigma_{k} u_{k}-Q_{k}(u) u_{k}+P_{k}(u) u_{k+r}+U_{k+r}(u, v), \\
\dot{v}_{l}=\sum_{\nu=1}^{s} b_{l \nu} v_{\nu}+V_{l}(u, v), \quad(k=1, \cdots r ; l=1, \cdots, s)
\end{gathered}
$$

with

$$
P_{k}(u)=\frac{1}{2} \sum_{\mu=1}^{r} p_{k \mu}\left(u_{\mu}^{2}+u_{\mu+r}^{2}\right), \quad Q_{k}(u)=\frac{1}{2} \sum_{\mu=1}^{r} q_{k \mu}\left(u_{\mu}^{2}+u_{\mu+r}^{2}\right),
$$

where $b_{l \nu}, p_{k \mu}, q_{k \mu}$ are real constants, and $s \times s$ matr $x B=\left(b_{l \nu}\right)$ has no ergenvalue that is an integer multiple of $\lambda_{1}$, and $U_{k}(u, v)$ and $V_{l}(u, v)$ and $C^{4}$ functions of $u_{1}, \cdots, u_{2 r}, v_{1}, \cdots, v_{s}$ such that

$$
\begin{gathered}
U_{k}\left(u, v^{2}\right)=O\left((|u|+|v|)^{4}\right), \quad V_{l}\left(u, v^{2}\right)=O\left((|u|+|v|)^{3}\right), \\
(k=1, \cdots, 2 r ; l=1, \cdots, s) .
\end{gathered}
$$

Here, $\left(u, v^{2}\right)=\left(u_{1}, \cdots, u_{2 r}, v_{1}^{2}, \cdots, v_{s}^{2}\right)$.

Proof. At first, we note that a substitution $x=\phi(w)$, which is invertible near the origin, takes the system (1.1) into

$$
\dot{w}=\phi_{w}^{-1} f(\phi(w)),
$$

where $\phi_{w}$ denotes the Jacobian matrix of $\phi$. Let $\tilde{f}(x)$ be a vector whose components $\tilde{f}_{k}(x)(k=1, \cdots, m)$ are cubic polynomials such that

$$
f_{k}(x)-\tilde{f}_{k}(x)=O\left(|x|^{4}\right) .
$$

Instead of (1.1), let us consider a system 


$$
x=\tilde{f}(x) .
$$

If there exists a substitution which takes (2.5) into the normal form (2.2) with the origin fixed, then the substitution also takes (1.1) into the normal form (2.2). Therefore, it suffices to determine the substitution (2.1) so that the system (2.5) is taken into the normal form (2.2).

By the assumptions, after a preliminary real linear substitution we may assume that $C=f_{x}(0)=\tilde{f}_{x}(0)$ has the form

$$
C=\left[\begin{array}{ll}
A & 0 \\
0 & B
\end{array}\right]
$$

with

$$
A=\left[\begin{array}{rr}
0 & D \\
-D & 0
\end{array}\right], \quad D=\operatorname{diag}\left(\sigma_{1}, \cdots, \sigma_{r}\right),
$$

where diag $\left(\sigma_{1}, \cdots, \sigma_{r}\right)$ denotes an $r \times r$ diagonal matrix with diagonal components $\sigma_{1}, \cdots, \sigma_{r}$, and $B$ is an $s \times s$ invertible matrix of which no eigenvalue is an integer multiple of $\lambda_{1}$.

Next we shall normalize the quadratic and cubic terms of $\tilde{f}_{k}(x)(k=1, \cdots, m)$ by a nonlinear substitution. To this end, it is better to work with complex form. At first we carry out a complex linear substitution

$$
x=T z
$$

with

$$
T=\left[\begin{array}{cc}
J & 0 \\
0 & I_{s}
\end{array}\right], \quad J=\frac{1}{\sqrt{2}}\left[\begin{array}{cc}
I_{r} & I_{r} \\
i I_{r} & -i I_{r}
\end{array}\right],
$$

where $I_{\jmath}(\jmath=r, s)$ is the $j \times j$ identity matrix.

Then the system (2.5) is transformed into

$$
\dot{z}=g(z)
$$

with

$$
g(z)=T^{-1} \tilde{f}(T z)=\left(5 z+g^{(2)}+g^{(3)}\right.
$$

where

$$
\mathfrak{S}=\left[\begin{array}{ll}
\mathfrak{A} & 0 \\
0 & B
\end{array}\right], \quad \mathfrak{A}=\operatorname{diag}\left(\lambda_{1}, \cdots, \lambda_{2 r}\right),
$$

and $g^{(\jmath)}(\jmath=2,3)$ are column vectors with entries $g_{1}^{(j)}, \cdots, g_{m}^{(\jmath)}$ which are homogeneous polynomials in $z_{1}, \cdots, z_{m}$ of degree $\jmath$.

Now we neglect the reality requirement on $f(x)$, and we will seek a complex nonlinear substitution $z=\varphi(\xi, \eta)$ which takes (2.10) into the following complex normal form: 


$$
\dot{\xi}_{k}=\lambda_{k} \xi_{k}+\left(\sum_{\mu=1}^{r} \tau_{k \mu} \xi_{\mu} \xi_{\mu+r}\right) \xi_{k}+X_{k}(\xi, \eta) \quad(k=1, \cdots, 2 r)
$$

$$
\dot{\eta}_{l}=\sum_{\nu=1}^{s} b_{l \nu} \eta_{\nu}+Y_{l}(\xi, \eta) \quad(l=1, \cdots, s),
$$

where $\tau_{k \mu}$ are complex constants, and $X_{k}(\xi, \eta)(k=1, \cdots, 2 r)$ and $Y_{l}(\xi, \eta) \quad(l=1$, $\cdots, s)$ are convergent power series in $\xi_{1}, \cdots, \xi_{2 r}, \eta_{1}, \cdots, \eta_{s}$ such that $X_{k}\left(\xi, \eta^{2}\right)$ contains terms of order $\geqq 4$ only, and $Y_{l}\left(\xi, \eta^{2}\right)$ contains terms of order $\geqq 3$ only.

For this purpose, let us restrict ourselves to the substitution of the form

$$
z=\varphi(\zeta)=\zeta+\varphi^{(2)}+\varphi^{(3)},
$$

where $\zeta$ is a column vector with $m$ entries $\zeta_{k}=\xi_{k}(k=1, \cdots, 2 r), \zeta_{l+2 r}=\eta_{l}(l=1$, $\cdots, s)$, and $\varphi^{(j)}(j=2,3)$ are column vectors with entries $\varphi_{1}^{(j)}, \cdots, \varphi_{m}^{(j)}$ which are homogeneous polynomials in $\zeta_{1}, \cdots, \zeta_{m}$ of degree $j$.

Our aim is to solve the system of functional equations

$$
g(\varphi(\zeta))=\varphi_{\zeta} h(\zeta)
$$

for cubic polynomials $\varphi_{k}(\zeta)=\zeta_{k}+\varphi_{k}^{(2)}+\varphi_{k}^{(3)}$ and power series $h_{k}(\zeta)(k=1, \cdots, m)$ so that the column vector $h(\zeta)$ has the form of the right-hand side of (2.13), in other words $h_{k}(\zeta)(k=1, \cdots, m)$ satisfy the following conditions :

[C.1] $\quad h_{k}(\zeta)-\lambda_{k} \xi_{k}(k=1, \cdots, 2 \gamma)$ contains no term $\xi^{\alpha} \eta^{\beta}$ with $|\alpha|+2|\beta| \leqq 3$ besides the terms of the form $\xi_{k}\left(\xi_{\mu} \xi_{\mu+r}\right)(\mu=1, \cdots, r)$.

[C.2] $\quad h_{l+2 r}(\zeta)-\sum_{\nu=1}^{s} b_{l \nu} \eta_{\nu}(l=1, \cdots, s)$ contains no term $\xi^{\alpha} \eta^{\beta}$ with $|\alpha|+2|\beta| \leqq 2$.

where the notation

$$
\xi^{\alpha} \eta^{\beta}=\prod_{\mu=1}^{2 r} \xi_{\mu}^{\alpha} \prod_{\nu=1}^{s} \eta_{\nu}^{\beta_{\nu}}, \quad|\alpha|=\sum_{\mu=1}^{2 r} \alpha_{\mu}, \quad|\beta|=\sum_{\nu=1}^{s} \beta_{\nu}
$$

is used for $\alpha=\left(\alpha_{1}, \cdots, \alpha_{2 r}\right)$ and $\beta=\left(\beta_{1}, \cdots, \beta_{s}\right)$ whose components are nonnegative integers. Then the solution $\varphi(\zeta)$ will define our desired substitution.

Now we will solve the system (2.15) for $\varphi_{k}(\zeta)$ and $h_{k}(\zeta)(k=1, \cdots, m)$ by comparison of coefficients in (2.15). In order that the solution is uniquely determined, we impose the following additional conditions on $\varphi(\zeta)$ :

$$
\varphi_{k}(\zeta)(k=1, \cdots, 2 r) \text { contains terms } \xi^{\alpha} \eta^{\beta} \text { with }|\alpha|+2|\beta| \leqq 3 \text { only, }
$$
and contains no term of the form $\xi_{k}\left(\xi_{\mu} \xi_{\mu+r}\right)(\mu=1, \cdots, r)$.

[C.4] $\varphi_{l+2 r}(\zeta)(l=1, \cdots, s)$ contains terms $\xi^{\alpha} \eta^{\beta}$ with $|\alpha|+2|\beta| \leqq 2$ only .

Comparing the linear terms in (2.15), $h(\zeta)$ must have the form 


$$
h(\zeta)=\left(\zeta \zeta+\sum_{j=2}^{\infty} h^{(j)},\right.
$$

where $h^{(j)}(j=2,3, \cdots)$ are again column vectors with entries $h_{1}^{(j)}, \cdots, h_{m}^{(j)}$ which are homogeneous polynomials in $\zeta_{1}, \cdots, \zeta_{m}$ of degree $j$. In what follows, for $y(\xi, \eta)$ a polynomial (or a power series) in $\xi_{1}, \cdots, \xi_{2 r}, \eta_{1}, \cdots, \eta_{s}$, the coefficient of $\xi^{\alpha} \eta^{\beta}$ in $y(\xi, \eta)$ will be denoted by $\{y\}_{\alpha \beta}$. We will determine $\left\{\varphi_{k}\right\}_{\alpha \beta}$ and $\left\{h_{k}\right\}_{\alpha \beta}$ $(k=1, \cdots, m)$ for $|\alpha|+|\beta| \geqq 2$.

The comparison of the quadratic terms in (2.15) leads us to the relation

$$
h^{(2)}+\varphi_{\xi}^{(2)}\left(\zeta-\left(\zeta \varphi^{(2)}=g^{(2)},\right.\right.
$$

which is represented in each component by

$$
F_{k}\left(h^{(2)}, \varphi^{(2)}\right)=g_{k}^{(2)} \quad(k=1, \cdots, m)
$$

with

$$
F_{k}\left(h^{(2)}, \varphi^{(2)}\right)=\left\{\begin{array}{r}
h_{k}^{(2)}+\sum_{\mu=1}^{2 r} \varphi_{k, \xi}^{(2)} \lambda_{\mu} \xi_{\mu}+\sum_{\mu=1}^{s} \varphi_{k, \eta_{\mu}}^{(2)}\left(\sum_{\nu=1}^{s} b_{\mu \nu} \eta_{\nu}\right)-\lambda_{k} \varphi_{k}^{(2)} \\
(k=1, \cdots, 2 r), \\
h_{k}^{(2)}+\sum_{\mu=1}^{2 r} \varphi_{k, \xi_{\mu}}^{(2)} \lambda_{\mu} \xi_{\mu}+\sum_{\mu=1}^{s} \varphi_{k, \eta_{\mu}}^{(2)}\left(\sum_{\nu=1}^{s} b_{\mu \nu} \eta_{\nu}\right)-\sum_{\mu=1}^{s} b_{k^{\prime}} \varphi_{\mu+2 r}^{(2)} \\
\left(k^{\prime}=k-2 r ; k=2 r+1, \cdots, m\right) .
\end{array}\right.
$$

We compare the coefficients of $\xi^{\alpha} \eta^{\beta}$ in (2.16). First we consider the case when $|\alpha|=2$ and $|\beta|=0$. In this case, since $\left\{h_{k}^{(2)}\right\}_{\alpha \beta}=0(k=1, \cdots, m)$ by the conditions [C.1] and [C.2], the comparison gives

where

$$
\left(\langle\alpha, \lambda\rangle I-(5)\left\{\varphi^{(2)}\right\}_{\alpha \beta}=\left\{g^{(2)}\right\}_{\alpha \beta},\right.
$$

$$
\langle\alpha, \lambda\rangle=\sum_{\mu=1}^{2 r} \alpha_{\mu} \lambda_{\mu}
$$

and $\left\{\varphi^{(2)}\right\}_{\alpha \beta}$ and $\left\{g^{(2)}\right\}_{\alpha \beta}$ are column vectors with $m$ entries $\left\{\varphi_{k}^{(2)}\right\}_{\alpha \beta}$ and $\left\{g_{k}^{(2)}\right\}_{\alpha \beta}$ $(k=1, \cdots, m)$ respectively, and $I$ is the $m \times m$ identity matrix. Here we have $\operatorname{det}\left(\langle\alpha, \lambda\rangle I-(5) \neq 0\right.$ because $\langle\alpha, \lambda\rangle-\lambda_{k} \neq 0$ for any $k=1, \cdots, m$ by the assumptions [A.1] and [A.2]. Therefore $\left\{\varphi^{(2)}\right\}_{\alpha \beta}$ is uniquely determined. Next we consider the case when $|\alpha|=|\beta|=1$. In this case, we must have $\xi^{\alpha} \eta^{\beta}=\xi_{\mu} \eta_{\nu}$ for some $\mu, \nu(\mu=1, \cdots, 2 r ; \nu=1, \cdots, s)$, and then we write $\left\{g_{k}^{(2)}\right\}_{\mu \nu},\left\{\varphi_{k}^{(2)}\right\}_{\mu \nu},\left\{h_{k}^{(2)}\right\}_{\mu \nu}$ in place of $\left\{g_{k}^{(2)}\right\}_{\alpha \beta},\left\{\varphi_{k}^{(2)}\right\}_{\alpha \beta},\left\{h_{k}^{(2)}\right\}_{\alpha \beta}$ respectively. For $k=1, \cdots, 2 r$, since $\left\{h_{k}^{(2)}\right\}_{\mu \nu}=0$ by the condition [C.1], the comparison gives

$$
\left(\lambda_{\mu}-\lambda_{k}\right)\left\{\varphi_{k}^{(2)}\right\}_{\mu \nu}+\sum_{l=1}^{s}\left\{\varphi_{k}^{(2)}\right\}_{\mu l} b_{l \nu}=\left\{g_{k}^{(2)}\right\}_{\mu \nu},
$$

or in vector form 


$$
\left(\left(\lambda_{\mu}-\lambda_{k}\right) I+{ }^{t} B\right)\left\{\varphi_{k}^{(2)}\right\}_{\mu}=\left\{g_{k}^{(2)}\right\}_{\mu} \quad(\mu=1, \cdots, 2 r),
$$

where $\left\{\varphi_{k}^{(2)}\right\}_{\mu}$ and $\left\{g_{k}^{(2)}\right\}_{\mu}$ are column vectors with $s$ entries $\left\{\varphi_{k}^{(2)}\right\}_{\mu \nu}$ and $\left\{g_{k}^{(2)}\right\}_{\mu \nu}$ $(\nu=1, \cdots, s)$ respectively, and $I$ is the $2 r \times 2 r$ identity matrix and ${ }^{t} B$ is the transposed matrix of $B$. Then these equations determine $\left\{\varphi_{k}^{(2)}\right\}_{\mu}(\mu=1, \cdots, 2 r)$ uniquely. On the other hand, for $k=2 r+1, \cdots, m$, we have $\left\{\varphi_{k}^{(2)}\right\}_{\mu \nu}=0$ by the condition [C.4], and it follows that $\left\{h_{k}^{(2)}\right\}_{\mu \nu}=\left\{g_{k}^{(2)}\right\}_{\mu \nu}(\mu=1, \cdots, 2 r ; \nu=1, \cdots, s)$. Finally, in the case when $|\alpha|=0$ and $|\beta|=2$, we have $\left\{\varphi_{k}^{(2)}\right\}_{\alpha \beta}=0(k=1, \cdots, m)$ by the conditions [C.3] and [C.4], and it follows that $\left\{h_{k}^{(2)}\right\}_{\alpha \beta}=\left\{g_{k}^{(2)}\right\}_{\alpha \beta}$. Thus the coefficients $\left\{\varphi_{k}\right\}_{\alpha \beta}$ and $\left\{h_{k}\right\}_{\alpha \beta}(k=1, \cdots, m)$ have been determined uniquely for $|\alpha|+|\beta|=2$.

Next, similarly the comparison of the cubic terms in (2.15) leads us to the relations

$$
\begin{array}{r}
F_{k}\left(h^{(3)}, \varphi^{(3)}\right)=g_{k}^{(3)}-\sum_{\mu=1}^{m} \varphi_{k, \xi_{\mu}}^{(2)} h_{\mu}^{(2)}+\text { cubic terms in } g_{k}^{(2)}(\varphi(\zeta)) \\
(k=1, \cdots, m),
\end{array}
$$

where the left-hand sides are cubic polynomials obtained by replacing $h_{k}^{(2)}$ and $\varphi_{1}^{(2)}, \cdots, \varphi_{m}^{(2)}$ in $F_{k}\left(h^{(2)}, \varphi^{(2)}\right)$ with $h_{k}^{(3)}$ and $\varphi_{1}^{(3)}, \cdots, \varphi_{m}^{(3)}$ respectively. We note that the right-hand sides are the cubic polynomials in which coefficients have been already known. If $|\beta| \neq 0$ or $k=2 r+1, \cdots, m$, we have $\left\{\varphi_{k}^{(3)}\right\}_{\alpha \beta}=0$ by the conditions [C.3] and [C.4], and then the corresponding coefficients $\left\{h_{k}^{(3)}\right\}_{\alpha \beta}$ are uniquely determined from (2.18). Therefore we consider the relations $(2.18)$ for $k=1, \cdots, 2 r$ only, and we will compare the coefficients of $\xi^{\alpha} \eta^{\beta}$ with $|\alpha|=3$ and $|\beta|=0$ in the both sides. Noting that $h_{k}^{(2)}(k=1, \cdots, m)$ contains no term $\xi^{\alpha} \eta^{\beta}$ with $|\alpha|=2$ and $|\beta|=0$, the comparison gives

$$
\left\{h_{k}^{(3)}\right\}_{\alpha \beta}+\left(\langle\alpha, \lambda\rangle-\lambda_{k}\right)\left\{\varphi_{k}^{(3)}\right\}_{\alpha \beta}=\left\{g_{k}^{(3)}\right\}_{\alpha \beta}+\left\{g_{k}^{(2)}(\varphi(\zeta))\right\}_{\alpha \beta} \quad(k=1, \cdots, 2 r) .
$$

Here we note that the right-hand sides are already known. By the assumption [A.2] with [A.1], we have $\langle\alpha, \lambda\rangle-\lambda_{k}=0$ if and only if $\alpha_{k}=\alpha_{\mu}=\alpha_{\mu+r}=1$ for some $\mu(\mu \neq k, \mu+r \neq k ; 1 \leqq \mu \leqq r)$, or $\alpha_{k}=2$ and $\alpha_{k+r}=1(1 \leqq k \leqq r), \alpha_{k-r}=1(r+1 \leqq k \leqq 2 r)$. In these cases, $\left\{h_{k}^{(3)}\right\}_{\alpha \beta}$ are uniquely determined from (2.19) and we have $\left\{\varphi_{k}^{(3)}\right\}_{\alpha \beta}$ $=0$ by the condition [C.3]. Otherwise we have $\left\{h_{k}^{(3)}\right\}_{\alpha \beta}=0$ by the condition [C.1] and then $\left\{\varphi_{k}^{(3)}\right\}_{\alpha \beta}$ are uniquely determined from (2.19). Thus the coefficients $\left\{\varphi_{k}\right\}_{\alpha \beta}$ and $\left\{h_{k}\right\}_{\alpha \beta}(k=1, \cdots, m)$ have been determined uniquely for $|\alpha|+|\beta|=3$.

We have now uniquely determined $\varphi(\zeta)$ of the form (2.14), and then $h(\zeta)$ is also uniquely determined so that (2.15) holds and the $h(\zeta)$ satisfies the conditions [C.1] and [C.2]. Therefore this $\varphi(\zeta)$ defines a substitution taking the system (2.10) into the complex normal form (2.13).

From now on, we shall take the reality of $f(x)$ into account, and investigate the effect of it on the above determined $\varphi(\zeta)$ and $h(\zeta)$. By complex conjugation of coefficients in the system (2.15) satisfied by the above determined $\varphi(\zeta)$ and $h(\zeta)$, and by setting $K=T^{-1} \bar{T}$, we have

$$
T^{-1} \tilde{f}(T K \bar{\varphi}(\zeta))=K \bar{\varphi} \zeta \bar{h}(\zeta),
$$


where $\bar{T}$ is the matrix obtained by complex conjugation of components of $T$, and $\bar{\varphi}(\zeta)$ and $\bar{h}(\zeta)$ are column vectors whose entries are obtained by complex conjugation of coefficients in $\varphi_{k}(\zeta)$ and $h_{k}(\zeta)(k=1, \cdots, m)$ respectively with the indeterminates $\zeta_{1}, \cdots, \zeta_{m}$ remaining fixed. Since we have

$$
K=\left(\begin{array}{ccc}
0 & I_{r} & \\
I_{r} & 0 & 0 \\
\hdashline 0 & & I_{s}
\end{array}\right),
$$

it follows that $K^{-1}=\bar{K}=K$. Therefore, if $\zeta$ is replaced by $K \zeta$, we have an identity

$$
g\left(\varphi^{*}(\zeta)\right)=\varphi_{\zeta}^{*} h^{*}(\zeta)
$$

where $\varphi^{*}(\zeta)=K \bar{\varphi}(K \zeta)$ and $h^{*}(\zeta)=K \bar{h}(K \zeta)$. This means that $\varphi^{*}(\zeta)$ and $h^{*}(\zeta)$ also satisfy (2.15). Furthermore, we can easily see that $\varphi^{*}(\zeta)$ and $h^{*}(\zeta)$ have the linear terms of the form $\zeta$ and $5 \zeta$ respectively and fulfill our four conditions [C.1], [C.2], [C.3] and [C.4]. Therefore it follows from the established uniqueness that

$$
\varphi(\zeta)=\varphi^{*}(\zeta), \quad h(\zeta)=h^{*}(\zeta)
$$

From the first identity, we see that $T \varphi(\zeta)$ is real if $\bar{\zeta}=K \zeta$. Let us introduce a substitution $\zeta=T^{-1} w$ with $w_{k}=u_{k}(k=1, \cdots, 2 r), w_{l+2 r}=v_{l} \quad(l=1, \cdots, s)$, then $T \varphi\left(T^{-1} w\right)$ is real if $w$ is real. Hence $x=T \varphi\left(T^{-1} w\right)$ defines a real analytic substitution. Moreover, from the second identity we have

$$
\tau_{k+r \mu}=\bar{\tau}_{k \mu} \quad(k, \mu=1, \cdots, r)
$$

in the complex normal form (2.13). Therefore, setting $\tau_{k \mu}=p_{k \mu}+\imath q_{k \mu}$, where $p_{k \mu}$ and $q_{k \mu}$ are real constants, this substitution $x=T \varphi\left(T^{-1} w\right)$ transforms the system (2.5) with (2.6) and (2.7) into the real normal form (2.2). Thus we have obtained the desired substitution (2.1) as the composition of the preliminary linear substitution and this nonlinear substitution $x=T \varphi\left(T^{-1} w\right)$. This completes the proof.

The substitution (2.1) which takes the system (1.1) into the normal form does not exist uniquely, that is to say, in the system (2.2) the coefficients $p_{k \mu}, q_{k \mu}$ are not uniquely determined. However, we can say that to some extent these coefficients are determined uniquely. This is due to the following lemma.

LEMMA 2. Consider the system (1.1) under the same assumptions of Lemma 1. Let (2.1) be a substitution taking the system (1.1) into the normal form (2.2) with (2.3) and (2.4). Suppose that another substitution

$$
x=\phi^{\prime}(u, v), \quad(u, v)=\left(u_{1}, \cdots, u_{2 r}, v_{1}, \cdots, v_{s}\right)
$$

takes the system (1.1) into the normal form (2.2) with the coefficients $b_{l \nu}^{\prime}, p_{k \mu}^{\prime}, q_{k \mu}^{\prime}$ in place of $b_{l \nu}, p_{k \mu}, q_{k \mu}$ respectuvely. Then the coefficients $p_{k \mu}, q_{k \mu}$ and $p_{k \mu}^{\prime}, q_{k \mu}^{\prime}$ satisfy the relations 


$$
\left(p_{1 \mu}^{\prime}, \cdots, p_{r \mu}^{\prime}, q_{1 \mu}^{\prime}, \cdots, q_{r \mu}^{\prime}\right)=\rho_{\mu}\left(p_{1 \mu}, \cdots, p_{r \mu}, q_{1 \mu}, \cdots, q_{r \mu}\right)
$$

where $\rho_{\mu}$ are positive scalars.

$$
(\mu=1, \cdots, r) \text {, }
$$

Proof. The substitution

$$
w=\phi^{-1} \circ \phi^{\prime}\left(w^{\prime}\right), \quad w=(u, v), \quad w^{\prime}=\left(u^{\prime}, v^{\prime}\right)
$$

takes the system (2.2) into another one of this normal form with the coefficients $b_{l \nu}^{\prime}, p_{k \mu}^{\prime}, q_{k \mu}^{\prime}$ in place of $b_{l \nu}, p_{k \mu}, q_{k \mu}$ respectively. This can be regarded as the composition of a linear change of variables $w$ into $w^{\prime \prime}=\left(u^{\prime \prime}, v^{\prime \prime}\right)$ and a nonlinear substitution of the form

$$
w^{\prime \prime}=\phi\left(w^{\prime}\right)=w^{\prime}+\phi^{(2)}\left(w^{\prime}\right)+\psi^{(3)}\left(w^{\prime}\right)+O\left(\left|w^{\prime}\right|^{4}\right),
$$

where $\psi^{(j)}\left(w^{\prime}\right)(j=2,3)$ are column vectors whose entries are homogeneous polynomials in $u_{1}, \cdots, u_{2 r}, v_{1}, \cdots, v_{s}$ of degree $j$. Then, by comparing the linear terms in these two systems of the same form, this linear substitution must have the form

$$
\begin{aligned}
& u_{k}=a_{k} u_{k}^{\prime \prime}+d_{k} u_{k+r}^{\prime \prime}, \quad u_{k+r}=-d_{k} u_{k}^{\prime \prime}+a_{k} u_{k+r}^{\prime \prime} \quad(k=1, \cdots, r), \\
& v=L v^{\prime \prime},
\end{aligned}
$$

where $a_{k}, d_{k}$ are real constants such that $a_{k}^{2}+d_{k}^{2} \neq 0$, and $L$ is an $s \times s$ non-singular matrix. Consequently, this linear substitution also takes the system (2.2) into the system of the same form, and the coefficients $p_{k \mu}, q_{k \mu}$ are replaced by

$$
p_{k \mu}^{\prime \prime}=\left(a_{\mu}^{2}+d_{\mu}^{2}\right) p_{k}, \quad q_{k \mu}^{\prime \prime}=\left(a_{\mu}^{2}+d_{\mu}^{2}\right) q_{k \mu} \quad(k, \mu=1, \cdots, r)
$$

respectively, and the matrix $B=\left(b_{l \nu}\right)$ is replaced by $B^{\prime \prime}=L^{-1} B L$. Furthermore, we can see that these coefficients $p_{k \mu}^{\prime \prime}$ and $q_{k \mu}^{\prime \prime}$ are not changed by the substitution of the form (2.22). To this end, let us consider the system of functional equations (2.15) under the assumption that not only $h(\zeta)$ but also $g(\zeta)$ has the right-hand side of the complex normal form (2.13). If $\varphi(\zeta)$ has the form (2.14), one is again led to the relations (2.19) by comparison of coefficients of $\xi^{\alpha} \eta^{\beta}$ with $|\alpha|=3$ and $|\beta|=0$. Since $g(\zeta)$ satisfies the same condition as [C.1], we have $\left\{g_{k}^{(2)}(\varphi(\zeta))\right\}_{\alpha \beta}$ $=0(k=1, \cdots, 2 r)$. Therefore, we have $\left\{g_{k}^{(3)}\right\}_{\alpha \beta}=\left\{h_{k}^{(3)}\right\}_{\alpha \beta}$ when $\langle\alpha, \lambda\rangle-\lambda_{k}=0$ $(k=1, \cdots, 2 r)$. Then it has been already proved that if the substitution of the form (2.14) takes the system (2.13) into another one of this complex normal form, the coefficients $\tau_{k \mu}(k=1, \cdots, 2 r ; \mu=1, \cdots, r)$ are not changed. This shows that if the substitution of the form (2.22) takes the above system of the form (2.2) into another one of this normal form, the coefficients $p_{k \mu}^{\prime \prime}$ and $q_{k \mu}^{\prime \prime}$ are not changed. Hence we have $p_{k \mu}^{\prime \prime}=p_{k \mu}^{\prime}, q_{k \mu}^{\prime \prime}=q_{k \mu}^{\prime}$. Therefore, setting $\rho_{\mu}=a_{\mu}^{2}+d_{\mu}^{2}$, we obtain the relations $(2.21)$.

Now we will study the normal form under the existence of an integral. Our aim is to prove the following lemma. 
LEMMA 3. Assume that the normal form (2.2) (with (2.3) and (2.4)) possesses an integral $\hat{G}(w)$ with $\hat{G}_{w}(0)=0$ which is of class $C^{5}$ near $w=0$, where $w=(u, v)$ and $\hat{G}_{w}(0)$ denotes the gradient vector at $w=0$. Then the Hessian matrix $\hat{G}_{w w}(0)$ must have the form

$$
\hat{G}_{w w}(0)=\left(\begin{array}{cc:c}
S_{11} & 0 & 0 \\
0 & S_{11} & \\
\hdashline 0 & S_{2}
\end{array}\right), \quad S_{11}=\operatorname{diag}\left(s_{1}, \cdots, s_{r}\right),
$$

where $s_{1}, \cdots, s_{r}$ are real constants and $S_{2}$ is a $(m-2 r) \times(m-2 r)$ matrix. Further, if $s_{k} \neq 0$ in (2.23), then $p_{k k}=0$ in (2.3) $(k=1, \cdots, r)$.

Proof. Let $\hat{f}(w)$ denote the right-hand side of the normal form (2.2). Since $\hat{G}(w)$ is an integral for (2.2), an identity

$$
\left\langle\hat{G}_{w}(w), \hat{f}(w)\right\rangle=0
$$

holds near the origin, where $\langle\cdot, \cdot\rangle$ denotes the usual scalar product. This identity includes $\langle S w, C w\rangle=0$, where $S=\hat{G}_{w w}(0)$ and $C$ is the matrix (2.6) with (2.7). Then we obtain the identity

$$
\langle S C w, w\rangle=0,
$$

which implies that $S C$ is alternating, namely

$$
{ }^{t}(S C)={ }^{t} C S=-S C \text {. }
$$

If we set

$$
S=\left[\begin{array}{cc}
S_{1} & S_{3} \\
{ }^{t} S_{3} & S_{2}
\end{array}\right],
$$

where $S_{1}$ and $S_{2}$ are $2 r \times 2 r$ and $(m-2 r) \times(m-2 r)$ symmetric matrices respectively, and $S_{3}$ is a $2 r \times(m-2 r)$ matrix, then it follows from (2.25) that

$$
\begin{aligned}
& A S_{1}=S_{1} A, \\
& A S_{3}=S_{3} B .
\end{aligned}
$$

Because any eigenvalue of $A$ and that of $B$ are distinct from each other, it follows from the second relation that $S_{3}=0$. Further, setting

$$
S_{1}=\left[\begin{array}{cc}
S_{11} & S_{13} \\
{ }^{t} S_{13} & S_{12}
\end{array}\right],
$$

where $S_{1,}(\jmath=1,2,3)$ are $r \times r$ matrices, then the above first relation is divided into

$$
D^{t} S_{13}=-S_{13} D
$$




$$
\begin{aligned}
& D S_{13}=-{ }^{t} S_{13} D, \\
& D S_{12}=S_{11} D .
\end{aligned}
$$

Then we can easily see that $S_{13}=0$ from the first and second relations, and noting that $S_{11}$ and $S_{12}$ are symmetric, we can also see from the third relation that $S_{11}$ and $S_{12}$ are diagonal and $S_{11}=S_{12}$. Let the diagonal components of $S_{11}$ be $s_{1}, \cdots, s_{r}$, then we obtain (2.23).

Again we consider the identity (2.24). Let us write $\hat{G}(w)$ and $\tilde{f}(w)$ as follows :

$$
\begin{aligned}
& \hat{G}(w)=\hat{G}(0)+\hat{G}^{(2)}(w)+\hat{G}^{(3)}(w)+\hat{G}^{(4)}(w)+O\left(|w|^{5}\right), \\
& \hat{f}(w)=\hat{f}^{(1)}(w)+\hat{f}^{(2)}(w)+\hat{f}^{(3)}(w)+O\left(|w|^{4}\right),
\end{aligned}
$$

where $\hat{G}^{(\jmath)}(w)(\jmath=2,3,4)$ are homogeneous polynomials in $u_{1}, \cdots, u_{2 r}, v_{1}, \cdots, v_{s}$ of degree $\jmath$, and $\hat{f}^{(j)}(w)(\jmath=1,2,3)$ are column vectors whose entries are homogeneous polynomials in $u_{1}, \cdots, u_{2 r}, v_{1}, \cdots, v_{s}$ of degree $\jmath$. Here we note that $\hat{G}^{(2)}(w)=(1 / 2)^{t} w S w$ and $\hat{f}^{(1)}(w)=C w$. From the identity (2.24), it follows that

$$
\sum_{\jmath=2}^{4}\left\langle\hat{G}_{w}^{(j)}(w), \hat{f}^{(5-\jmath)}(w)\right\rangle=0
$$

In order to consider this identity in complex form, we carry out the substitution $w=T \zeta$ with $(2.9)$ and $\zeta_{k}=\xi_{k}(k=1, \cdots, 2 r), \zeta_{l+2 r}=\eta_{l}(l=1, \cdots, s)$. Since $\hat{G}_{w}^{(j)}(w)=$ ${ }^{t}\left(T^{-1}\right) \hat{G}_{\zeta}^{(j)}(T \zeta)$, we have

$$
\sum_{j=2}^{4}\left\langle\hat{G}_{\zeta}^{(j)}(T \zeta), T^{-1} \hat{f}^{(5-\jmath)}(T \zeta)\right\rangle=0
$$

We note that

$$
\sum_{j=1}^{3} T^{-1} \hat{f}^{(\jmath)}(T \zeta)
$$

has the form of the right-hand side of complex normal form (2.13). Consequently, $\left\langle\hat{G}_{\zeta}^{(3)}(T \zeta), T^{-1} \hat{f}^{(2)}(T \zeta)\right\rangle$ contains no product in powers of $\xi_{k} \xi_{k+r}(k=1, \cdots, r)$ only. Further, in order that

$$
\left\langle\hat{G}_{\zeta}^{(4)}(T \zeta), T^{-1} \hat{f}^{(1)}(T \zeta)\right\rangle=\sum_{k=1}^{2 r} \hat{G}_{\xi_{k}}^{(4)}(T \zeta) \lambda_{k} \xi_{k}+\sum_{l=1}^{s} \hat{G}_{\eta_{l}^{(4)}}^{(4)}(T \zeta) \sum_{\nu=1}^{s} b_{l_{\nu}} \eta_{\nu}
$$

contains such a product, $\hat{G}^{(4)}(T \zeta)$ must contain such a product. However, since $\lambda_{k+r}=\bar{\lambda}_{k}=-\lambda_{k}$, it does not enter in the polynomial $\left\langle\hat{G}_{\zeta}^{(4)}(T \zeta), T^{-1} \hat{f}^{(1)}(T \zeta)\right\rangle$. Therefore, noting that $\hat{G}_{\zeta}^{(2)}(T \zeta)={ }^{t} T S T \zeta$, the coefficient of $\left(\xi_{k} \xi_{k+r}\right)\left(\xi_{\mu} \xi_{\mu+r}\right)$ in $\left\langle\hat{G}_{\zeta}^{(2)}(T \zeta)\right.$, $\left.T^{-1} \hat{f}^{(3)}(T \zeta)\right\rangle$ is

$$
s_{k}\left(\tau_{k \mu}+\tau_{k+r \mu}\right)+s_{\mu}\left(\tau_{\mu k}+\tau_{\mu+r k}\right)=0 .
$$

Thus, setting $k=\mu$, if $s_{k} \neq 0$ then we have $\tau_{k k}=-\tau_{k+r}=-\bar{\tau}_{k k}$, namely $p_{k k}=0$. This completes the proof. 


\section{The Existence Theorem}

In this section, we shall establish the existence of a one-parameter family of long periodic solutions of (1.1) near the origin. This will be done by imposing suitable conditions on the coefficients in $P_{k}(u)$ and $Q_{k}(u)(k=1, \cdots, r)$ in (2.3). Before stating the existence theorem, we present a lemma due to D.S. Schmidt [4], which is fundamental for our discussions.

Let the system (1.1) possess an integral $G(x)$ which is of class $C^{2}$ near the origin. We consider the one-parameter system

$$
\dot{x}=f(x)+\gamma G_{x}(x),
$$

where $\gamma$ is a real parameter. Then we have

LEMMA 4. If $\gamma \neq 0$, the system (3.1) has no periodic solution $x(t)$ such that $G_{x}(x(t)) \not \equiv 0$.

Proof. Let $x(t)$ be any solution of (3.1) for $\gamma \neq 0$. Then

$$
\frac{d}{d t} G(x(t))=\left\langle G_{x}(x(t)), f(x(t))+\gamma G_{x}(x(t))\right\rangle \text {. }
$$

Since $G(x)$ is an integral for (1.1), $\left\langle G_{x}(x), f(x)\right\rangle=0$ is satisfied identically, and then we have

$$
\frac{d}{d t} G(x(t))=\gamma\left|G_{x}(x(t))\right|^{2} .
$$

Therefore, if $\gamma \neq 0, G(x(t))$ is a monotone function of $t$ unless $G_{x}(x(t)) \equiv 0$. Then $x(t)$ can not be a periodic solution such that $G_{x}(x(t)) \not \equiv 0$. This proves the lemma.

Now we state our existence theorem.

THEOREM. Consider the system (1.1) near the equilibrum $x=0$. Let $f(x)$ be of class $C^{l}(l \geqq 4)$ near $x=0$, and let the system (1.1) possess an integral $G(x)$ with $G_{x}(0)=0$ which is of class $C^{l+1}$ near $x=0$. Assume that the eigenvalues of $C=$ $f_{x}(0)$ satisfy the assumptions [A.1] and [A.2], and that the Hessian $G_{x x}(0)$ restructed to $E_{1}$ is nondegenerate, where $E_{1}$ is the real two-dimensional eigenspace corresponding to $\lambda_{1}$ and $\lambda_{1+r}$. Moreover, assume that

$$
p_{k 1}^{2}+\left(q_{k 1}-n_{k} q_{11}\right)^{2} \neq 0 \quad(k=2,3, \cdots, r)
$$

in the normal form (2.2) obtained by a suntable substitution (2.1). Then there exists a family of periodic solutions of (1.1) which depends smoothly of class $C^{l-3}$ on a real parameter $\varepsilon$, with $\varepsilon=0$ corresponding to the equilibrum $x=0$, and whose primutive period $T(\varepsilon)$ is of class $C^{l-3}$ in $\varepsilon$ with $T(0)=2 \pi / \sigma_{1}$.

Remarks. (i) By Lemma 2, the assumption (3.2) is independent of the choice 
of the substitution taking (1.1) into the normal form (2.2).

(ii) If $f(x)$ and $G(x)$ are of class $C^{\infty}$ or real analytic, the above family of periodic solutions depends smoothly of class $C^{\infty}$ or analytically on a parameter $\varepsilon$.

Proof. By Lemma 1 and Lemma 3, we can bring the system (1.1) into the normal form (2.2) by a suitable substitution $x=\phi(w)$ with $w_{k}=u_{k}(k=1, \cdots, 2 r)$, $w_{l+2 r}=v_{l}(l=1, \cdots, s)$, and the system (2.2) possesses an integral $G(\phi(w))$ whose Hessian at the origin has the form (2.23). Let $\hat{f}(w)$ denote the right-hand side of (2.2) and $\hat{G}(w)$ denote the integral $G(\phi(w))$. Consider the one-parameter system

$$
\dot{w}=\hat{f}(w)+\gamma \hat{G}_{w}(w) .
$$

Our goal is to establish the existence of a family of periodic solutions of (3.3) along which $\hat{G}(w)$ is not stationary. Then, by Lemma 4 we must have $\gamma=0$ for these periodic solutions, and therefore they define a family of periodic solutions of (2.2).

By replacing the independent variable $t$ by $\sigma_{1} t$, we may assume $\sigma_{k}=n_{k}$ $(k=1, \cdots, r)$ in $(2.2)$, and then we seek periodic solutions of (3.3) with primitive periods near $2 \pi$. Since the desired periodic solutions are expected to be near the plane $v=0$, we introduce a stretching transformation $u \rightarrow \varepsilon u, v \rightarrow \varepsilon^{2} v$ with a small positive parameter $\varepsilon$. Further, introducing a stretching of the parameter $\gamma$ by $\gamma \rightarrow \varepsilon^{2} \gamma$, the system (3.3) is replaced by

$$
\begin{aligned}
& u_{k}=n_{k} u_{k+r}+\varepsilon^{2}\left(s_{k} \gamma u_{k}+P_{k}(u) u_{k}+Q_{k}(u) u_{k+r}\right)+O\left(\varepsilon^{3}\right), \\
& \dot{u}_{k+r}=-n_{k} u_{k}+\varepsilon^{2}\left(s_{k} \gamma u_{k+r}-Q_{k}(u) u_{k}+P_{k}(u) u_{k+r}\right)+O\left(\varepsilon^{3}\right), \\
& \dot{v}=B v+O(\varepsilon), \quad(k=1, \cdots, r) .
\end{aligned}
$$

Here we note that the right-hand sides of (3.4) can be considered as $C^{l-1}$ functions in $m+1$ variables $u_{1}, \cdots, u_{2 r}, v_{1}, \cdots, v_{s}$ and $\varepsilon$ in a neighborhood of the origin.

Our aim is to seek periodic solutions of (3.4) depending on the parameter $\varepsilon$ and corresponding values of $\gamma$. First we note that for $\varepsilon=0$ the system (3.4) has periodic solutions $(u(t), v(t))=(\exp (t A) u(0), 0)$, where $A$ is the matrix (2.7) with $\sigma_{k}=n_{k}$, and these periodic solutions have primitive period $2 \pi$ if $u_{1}(0) \neq 0$ or $u_{1+r}(0)$ $\neq 0$. Since we have

$$
\frac{d}{d t}\left\{u_{k}^{2}(t)+u_{k+r}^{2}(t)\right\}=O\left(\varepsilon^{2}\right)
$$

for any solution $(u(t), v(t))$ of (3.4), it follows that

$$
u_{k}^{2}(t)+u_{k+r}^{2}(t)=u_{k}^{2}(0)+u_{k+r}^{2}(0)+O\left(\varepsilon^{2}\right) \quad(k=1, \cdots, r) .
$$

Then, by integrating (3.4) we have

$$
u(t)=\exp \left[t\left\{A+\varepsilon^{2} \gamma\left[\begin{array}{ll}
S_{11} & 0 \\
0 & S_{11}
\end{array}\right]+\varepsilon^{2}\left[\begin{array}{rr}
P & Q \\
-Q & P
\end{array}\right]\right\}\right] u(0)+O\left(\varepsilon^{3}\right),
$$




$$
v(t)=\exp (t B) v(0)+O(\varepsilon),
$$

where

$$
P=\operatorname{diag}\left\{P_{1}(u(0)), \cdots, P_{r}(u(0))\right\}, \quad Q=\operatorname{diag}\left\{Q_{1}(u(0)), \cdots, Q_{r}(u(0))\right\} .
$$

For $(u(t), v(t))$ to be a periodic solution with period $\omega$ near $2 \pi$, it is necessary and sufficient that $u(\omega)=u(0)$ and $v(\omega)=v(0)$. Setting $\omega=2 \pi\left(1+\varepsilon^{2} \theta\right)$, this periodicity condition leads to

$$
\begin{aligned}
& 2 \pi \varepsilon^{2}\left[\left\{\theta A+\gamma\left[\begin{array}{ll}
S_{11} & 0 \\
0 & S_{11}
\end{array}\right]+\left[\begin{array}{rr}
P & Q \\
-Q & P
\end{array}\right]\right\} u(0)+O(\varepsilon)\right]=0, \\
& \{\exp (2 \pi B)-I\} v(0)+O(\varepsilon)=0 .
\end{aligned}
$$

Hence, replacing $u(0), v(0)$ by $u$, we have only to solve the system of $m$ equations

$$
\begin{aligned}
& \Gamma_{k}=\theta n_{k} u_{k+r}+\gamma s_{k} u_{k}+P_{k}(u) u_{k}+Q_{k}(u) u_{k+r}+O(\varepsilon)=0, \\
& \Gamma_{k+r}=-\theta n_{k} u_{k}+\gamma s_{k} u_{k+r}-Q_{k}(u) u_{k}+P_{k}(u) u_{k+r}+O(\varepsilon)=0, \\
& \Gamma=\{\exp (2 \pi B)-I\} v+O(\varepsilon)=0, \\
& (k=1, \cdots, r)
\end{aligned}
$$

for $\theta, \gamma, u_{1}, \cdots, u_{2 r}, v_{1}, \cdots, v_{s}$. Here $\Gamma_{k}(k=1, \cdots, 2 \gamma)$ and $\Gamma$ are $C^{l-3}$ functions in $\theta, \gamma, u_{1}, \cdots, u_{2 r}, v_{1}, \cdots, v_{s}$ and $\varepsilon$ in a neighborhood of the origin. To this end, we stipulate $u_{1}=u_{1+r}=1$ and then we will solve the implicit system (3.5) for $m$ unknowns $\theta, \gamma, u_{k}, u_{k+r}(k=2, \cdots, r), v_{1}, \cdots, v_{s}$. Since nondegeneracy of $G_{x x}(0)$ restricted to $E_{1}$ implies $s_{1} \neq 0$, we have $p_{11}=0$ by Lemma 3 . Then, for $\varepsilon=0$ the system (3.5) has a solution $\theta=-q_{11}, \gamma=0, u_{k}=u_{k+r}=v_{l}=0(k=2, \cdots, r ; l=1, \cdots, s)$, which corresponds to a periodic solution with primitive period $2 \pi$. With easy computation, the Jacobian at this point is given by

$$
\begin{aligned}
& \operatorname{det}\left\{\frac{\partial\left(\Gamma_{1}, \Gamma_{1+r}, \cdots \cdots, \Gamma_{r}, \Gamma_{2 r}, \Gamma\right)}{\partial\left(\theta, \gamma, u_{2}, u_{2+r}, \cdots, u_{r}, u_{2 r}, v\right)}\right\} \\
& =2 s_{1} \prod_{k=2}^{r}\left\{p_{k 1}^{2}+\left(q_{k 1}-n_{k} q_{11}\right)^{2}\right\} \times \operatorname{det}\{\exp (2 \pi B)-I\} .
\end{aligned}
$$

By the assumptions, this is a nonvanishing determinant. Then, by the implicit function theorem, in a sufficiently small neighborhood of $\varepsilon=0$, there exist $C^{l-3}$ functions $\theta(\varepsilon), \gamma(\varepsilon), u_{k}(\varepsilon), u_{k+r}(\varepsilon), v(\varepsilon)(k=2, \cdots, r)$ satisfying the system (3.5) with $u_{1}=u_{1+r}=1$. Hence the system (3.3) has a family of periodic solutions with primitive periods $2 \pi\left(1+\varepsilon^{2} \theta(\varepsilon)\right)$, which depends smoothly of class $C^{l-3}$ on the parameter $\varepsilon$. Furthermore, these periodic solutions $w(t ; \varepsilon)=(u(t ; \varepsilon), v(t ; \varepsilon))$ satisfy

$$
\hat{G}_{u_{1}}(w(t ; \varepsilon))=s_{1} \varepsilon+O\left(\varepsilon^{2}\right) \neq 0
$$


for sufficiently small $\varepsilon \neq 0$, and therefore it follows that $\gamma(\varepsilon)=0$. This proves the existence of a desired family of periodic solutions of (2.2), i. e. of (1.1).

Remark. The periodic family whose existence has been established here consists of periodic solutions $w(t ; \varepsilon)=(u(t ; \varepsilon), v(t ; \varepsilon))$ such that

$$
\begin{array}{rlrl}
u_{1}(t ; \varepsilon)=O(\varepsilon), \quad u_{1+r}(t ; \varepsilon)=O(\varepsilon), & \\
u_{k}(t ; \varepsilon)=O\left(\varepsilon^{2}\right), \quad u_{k+r}(t ; \varepsilon)=O\left(\varepsilon^{2}\right) & & (k=2, \cdots, r), \\
v_{l}(t ; \varepsilon)=O\left(\varepsilon^{3}\right) & & (l=1, \cdots, s),
\end{array}
$$

together with $u_{1}(0 ; \varepsilon)=u_{1+r}(0 ; \varepsilon)=\varepsilon$. Therefore, we have

$$
\hat{G}(w(t ; \varepsilon))=G(0)+s_{1} \varepsilon^{2}+O\left(\varepsilon^{3}\right) .
$$

This means that for sufficiently small $\delta>0$, every integral surface $G(x)=\hat{G}(w)=$ $G(0)+s_{1} \delta$ contains one periodic orbit which belongs to the above periodic family.

\section{REFERENCES}

[1] J. Henrard, Lyapunov's Center Theorem for Resonant Equilibrium, J. Differential Equations 14 (1973), 431-441.

[2] J. Moser, Periodic Orbits near an Equilibrıum and a Theorem by Alan Weinstein, Comm. Pure Appl. Math. 29 (1976), 727-747.

[3] J. Moser, Addendum to "Perıodic Orbits near an Equilibrium and a Theorem by Alan Weinstein", Comm. Pure Appl. Math. 31 (1978), 529-530.

[4] D.S. Schмidt, Hopf's Birfurcation Theorem and the Center Theorem of Liapunov with Resonance Cases, J. Math. Anal. Appl. 63 (1978), 354-370.

[5] D. S. Schmidt and D. Sweet, A Unifyıng Theory in Determining Periodic Families for Hamiltonian Systems at Resonance, J. Differential Equations 14 (1973), 597-609.

[6] C.L. Siegel And J. Moser, Lectures on Celestial Mechanics, Springer-Verlag, 1971, in particular, $§ 16$.

Department of Mathematics

Tokyo Institute of Technology 